\title{
Perirhinal Cortex Contributes to Accuracy in Recognition Memory and Perceptual Discriminations
}

\author{
Edward B. 0’Neil, ${ }^{1}$ Anthony D. Cate, ${ }^{2}$ and Stefan Köhler ${ }^{1}$ \\ ${ }^{1}$ Department of Psychology, University of Western Ontario, London, Ontario N6A 5C2, Canada, and ${ }^{2}$ Human Cognitive Neurophysiology Laboratory, \\ Veterans Affairs Northern California Health Care System, Martinez, California 94553
}

The prevailing view of the medial temporal lobe (MTL) holds that its structures are dedicated to long-term declarative memory. Recent evidence challenges this position, suggesting that perirhinal cortex (PRc) in the MTL may also play a role in perceptual discriminations of stimuli with substantial visual feature overlap. Relevant neuropsychological findings in humans have been inconclusive, likely because studies have relied on patients with large and variable MTL lesions. Here, we conducted a functional magnetic resonance imaging study in healthy individuals to determine whether PRc shows a performance-related involvement in perceptual oddball judgments that is comparable to its established role in recognition memory. Morphed faces were selected as stimuli because of their large degree of feature overlap. All trials involved presentation of displays with three faces. The perceptual oddball task required identification of the face least similar to the other display members. The memory task involved forced-choice recognition of a previously studied face. When levels of behavioral performance were matched, we observed comparable levels of activation in right PRc for both tasks. Moreover, right PRc activity differentiated between accurate and inaccurate trials in both tasks. Together these results indicate that declarative memory demands are not a prerequisite for a performance-related engagement of PRc and that the introduction of such declarative memory demands in an otherwise closely matched perceptual task does not necessarily lead to an increase in PRc involvement. As such our findings show that declarative memory and perception are not as clearly separable at the level of MTL functioning as traditionally thought.

\section{Introduction}

It is firmly established that the integrity of the medial temporal lobe (MTL) is critical for memory functioning (Milner et al., 1998). The prevailing view is that the MTL, which consists of the hippocampus, entorhinal, perirhinal, and parahippocampal cortex, forms a system dedicated to declarative long-term memory (Squire et al., 2004). The MTL is widely connected with the neocortex; perirhinal cortex (PRc) receives much of its neocortical input from unimodal association areas, including significant contributions from the ventral visual stream (VVS) pertaining to object qualities (Suzuki and Amaral, 1994). In light of this connectivity, recent reports of visuoperceptual deficits in association with PRc damage have led some researchers to question the standard view of MTL functioning and the implied notion that a sharp boundary separates the MTL from the VVS (Buckley and Gaffan, 2006; Murray et al., 2007; Baxter, 2009). Instead, PRc may be best understood as an extension of the representational hierarchy within the VVS for object identification (Bussey and Saksida, 2007; Murray et al., 2007). According to such an account,

Received Jan. 22, 2009; revised April 27, 2009; accepted May 4, 2009.

This research was funded by Canadian Institutes for Health Research (CIHR) Operating Grants FRN 68933 and MOP 93644 (to S.K.). We thank Adam McLean, Jonathan Cant, and Teresa McAdam for assistance with data analysis and figure preparations. We are also grateful to Dr. Melvyn Goodale and other members of the CIHR Group for Action and Perception for several informative discussions of the data reported.

Correspondence should be addressed to Dr. Stefan Köhler, Department of Psychology, University of Western Ontario, London, Ontario N6A 5C2, Canada. E-mail: stefank@uwo.ca.

DOI:10.1523/JNEUROSCI.0374-09.2009

Copyright $\odot 2009$ Society for Neuroscience $\quad 0270-6474 / 09 / 298329-06 \$ 15.00 / 0$
PRc is recruited in tasks, perceptual or mnemonic, that require discriminations of objects with highly overlapping shape features, including but not restricted to faces. PRc may provide a representation of the conjunctions of features (Murray and Bussey, 1999) or of gestalt characteristics (Cate and Köhler, 2006), critical when individual shape features are insufficient for unique object identification. Such a higher-order representation is also beneficial for recognition of prior occurrence as a constant stream of visual input creates massive interference at the feature level, which needs to be resolved for the assessment of the familiarity of specific objects (Cowell et al., 2006).

Although a critical role for PRc in recognition memory is undisputed, its contributions beyond declarative memory remain highly controversial (Squire et al., 2004; Hampton, 2005; Suzuki, 2009). For example, two recent studies in neurological patients with lesions that included PRc provided conflicting results, despite highly similar experimental paradigms to tax online processing of objects in perceptual tasks (Lee et al., 2005a; Shrager et al., 2006). Furthermore, although imaging investigations in healthy individuals have begun to reveal an involvement of PRc in visuoperceptual tasks (Devlin and Price, 2007; Lee et al., 2008), it is presently unknown whether PRc activation is in fact related to accurate performance, and whether it is comparable to that seen in recognition memory. If representational factors dictate whether PRc is recruited, regardless of declarative memory demands, one would predict that its activity is related to accuracy on both types of tasks.

Here, we conducted a functional magnetic resonance imaging 
(fMRI) study to compare the role of PRc in recognition memory and perceptual oddball judgments. As in the aforementioned patient studies, morphed faces served as stimuli. Critically, we manipulated the difficulty of both tasks to allow for direct comparison at matched levels of accuracy. To investigate potential differences in the PRc response with that in more posterior VVS regions previously implicated in face processing, we also examined experimental effects in the fusiform face area (FFA) (Kanwisher et al., 1997).

\section{Materials and Methods}

Participants. Eighteen right-handed healthy subjects (10 male; age range, 23-29 years) participated in the study. All subjects had normal or corrected-to-normal vision and gave written informed consent. Participants received compensation for their participation. This study received approval from the Health Sciences Research Ethics Board at the University of Western Ontario.

Materials. Trials of both experimental tasks consisted of three artificially created faces (i.e., triplets) obtained by morphing two original color face photographs of Caucasian individuals with neutral facial expression. The two original faces of each trial-unique face pair were designated as endpoints on a morph continuum. Triplet members for the perception task were captured at different points on this continuum, such that the distance between the oddball image and its neighbor was larger than the distance between the other two images. To create three different levels of difficulty for the perception task, this increase in distance for the oddball was manipulated systematically (see supplemental Fig. 1 for additional details, available at www.jneurosci.org as supplemental material). Triplet members for the memory task were equally spaced along the morph continuum.

Additional stimuli were created for a luminance-baseline task that required no processing of visual shape features. Two squares of identical luminance and a third with a $5 \%$ increase were presented against a visual noise background. Luminance levels for these squares varied across trials.

Experimental procedure. The experiment included six fMRI runs in a fast event-related design. Each run was preceded by a study phase in which the 12 faces that served as targets in the memory task were presented for memorization. Faces were studied for $3000 \mathrm{~ms}$, with a $1000 \mathrm{~ms}$ intertrial interval. Half of the set of studied faces was presented once (difficult condition), whereas the other half was repeated three times (easy condition). During scanning, each trial began with a $1000 \mathrm{~ms}$ cue indicating the type of task to be performed. Each display of triplets was presented for $5000 \mathrm{~ms}$, followed by jittered fixation (Fig. 1). Trial order and jitter were optimized using the "OptSeq2" algorithm (http://surfer. nmr.mgh.harvard.edu/optseq/).

Every run included six trials from each difficulty level of the experimental tasks as well as the luminance-baseline task. Perception trials required selection of the face most different from the other two. Luminance trials required selection of the brightest square. Memory trials required selection of the face identical to one previously studied. Participants viewed the displays through a mirror at an approximate size of $22 \times 19^{\circ}$ visual angle. Responses were made using a keypad without performance feedback.

For investigation of responses in the FFA, the imaging protocol also included two functional localizer runs to identify this region. Here, we used the protocol described by Ganel et al. (2006), which involved presentation of face, object, and place stimuli in a blocked design under passive viewing instructions.

$f M R I$ data acquisition and image analysis. All fMRI scanning was completed on a 4 Tesla whole-body scanner (Varian; Siemens) using a custom head coil. To optimize signal in PRc, a localized second-ordershimming algorithm was combined with a multishot, short echo-timeimaging sequence. An oblique coronal orientation was selected for image acquisition to prevent inclusion of the eyes in the functional volume. The resulting volume captured the temporal and occipital lobe from the temporal pole to the occipital pole. For each subject, 19 contiguous $4 \mathrm{~mm}$ slices were obtained with a field of view of $22 \times 22 \mathrm{~cm}$ (sampled with a $64 \times 64$ matrix) and an in-plane resolution of $3.44 \times 3.44 \mathrm{~mm}$. A T2*

\section{Study (pre-scan)}

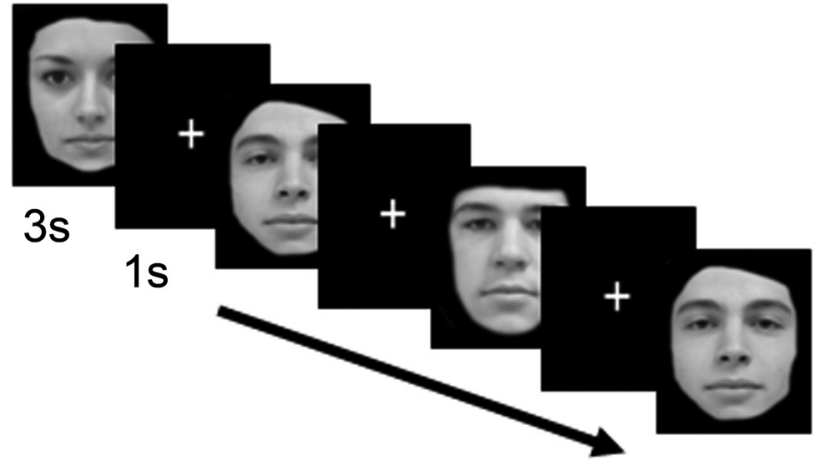

\section{Test (scanned)}

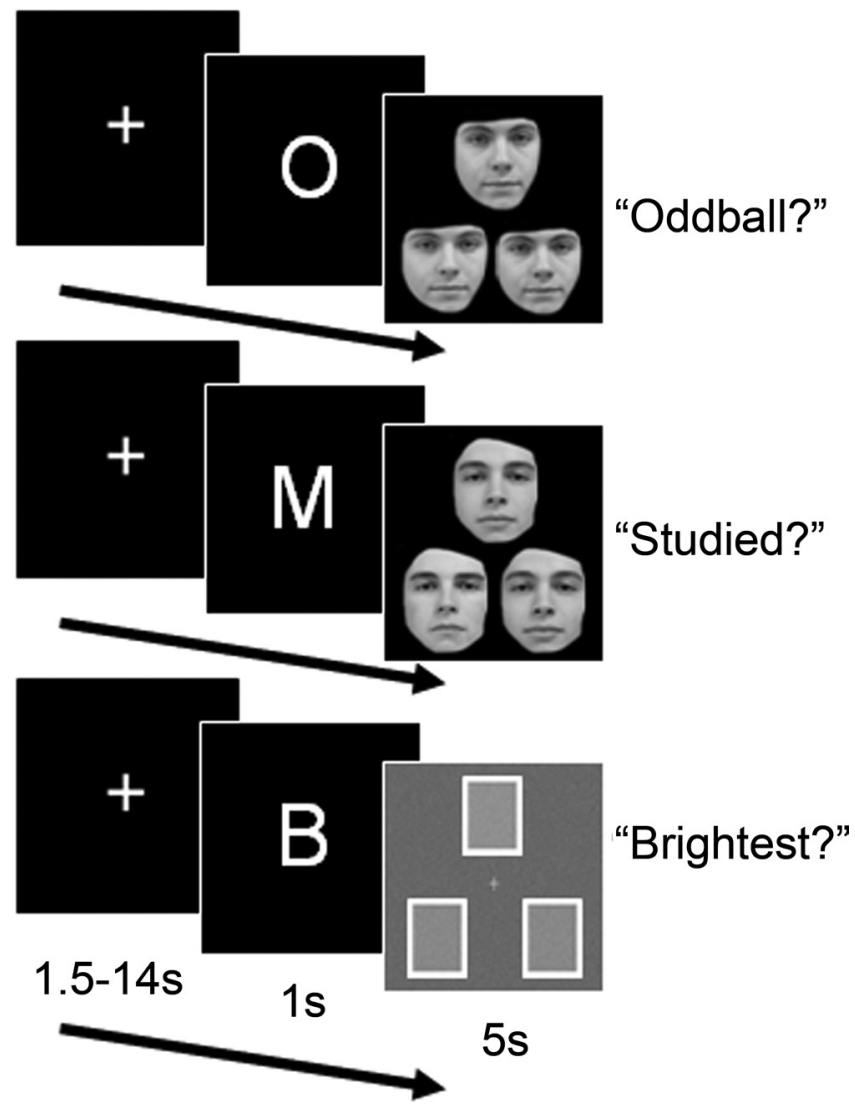

Figure 1. Experimental design. Each experimental run consisted of an initial study phase (prescanning) that required memorization of a series of faces. Under scanning, participants made perceptual oddball ( 0 ), forced-choice recognition memory (M), and luminance- baseline judgments (B).

weighted spiral image acquisition was used for all functional scans [echo time $(\mathrm{TE})=12 \mathrm{~ms}$; repetition time $(\mathrm{TR})=625 \mathrm{~ms}$; i.e., total volume acquisition time of $2500 \mathrm{~ms}$ with four-shot sequence; flip angle, $30^{\circ}$ ] with 160 volumes per run (150 volumes in the localizer runs). A T1-weighted high-resolution anatomical scan was acquired in the same plane as the functional images ( 144 slices; TR $=45 \mathrm{~ms}$; TE $=3 \mathrm{~ms} ; 256 \times 256$ matrix, in-plane resolution of $0.86 \times 0.86 \mathrm{~mm}$ with $1 \mathrm{~mm}$ slice thickness). $\mathrm{fMRI}$ data were analyzed using Brain Voyager QX 1.8 software (Brain Innovation). Functional images were resampled into $3 \mathrm{~mm}$ isotropic voxels, 
Table 1. Mean accuracy and response times for correct trials across participants

\begin{tabular}{lll}
\hline Condition & Accuracy (\% correct) & Response time (in ms) \\
\hline $\begin{array}{lll}\text { Memory } \\
\text { Difficult }\end{array}$ & \\
Easy & $54.60( \pm 2.72)$ & $3098.17( \pm 96.75)$ \\
Perception & $66.08( \pm 2.80)$ & $2926.08( \pm 89.81)$ \\
Difficult & & $3071.47( \pm 94.70)$ \\
Easy & $50.45( \pm 1.98)$ & $2865.71( \pm 72.35)$ \\
Baseline & $72.90( \pm 1.85)$ & $2233.22( \pm 124.00)$ \\
\hline Note that both acsuracy and response times were matched for the difficult memory and perception conditions
\end{tabular}

Note that both accuracy and response times were matched for the difficult memory and perception conditions (mean \pm SEM).

high-pass filtered, coregistered with the anatomical image, and transformed into standardized Talairach space. Resulting images were smoothed using a three-dimensional Gaussian kernel with a full-width at half maximum (FMHW) value of $6 \mathrm{~mm}$. Data were convolved with a double gamma hemodynamic response function and examined in a random-effects general linear model (GLM). Mean intensity of the volume was included as a covariate-of-no-interest.

A whole-volume voxelwise approach was used to identify PRc activation related to our experimental manipulations. Experimental effects exceeding a threshold of $p<0.001$ (uncorrected) were considered significant. For the conjunction analysis (Nichols et al., 2005), this significance level reflected the joint probability of the two independent contrasts included. Localization of activation in PRc was guided by published neuroanatomical protocols (Insausti et al., 1998). For purpose of comparison, the FFA was identified at the group level as the most anterior region in the right fusiform gyrus that showed greater activation for faces than places and greater activation for faces than objects ( $p<0.01$, uncorrected, for each contrast). One subject was unable to complete the functional localizer runs, and one run had to be discarded due to malfunction of the scanner.

\section{Results}

\section{Behavioral results}

Participants performed at above chance level of accuracy in each condition. Difficulty manipulations were effective for the memory task and for the perception task [memory, $t_{(1,17)}=-3.24$, $p<0.05$; perception, $\left.F_{(2,53)}=27.97, p<0.05\right]$. Closer inspection of data in the middle perception condition revealed, however, that while accuracy was approximately halfway in-between the difficult and easy conditions in terms of group mean, individual subjects' performance fell closer to levels for these extreme conditions than to their mean in the majority of cases. Consequently, for the fMRI analyses, we recoded the trials from the middle condition as "easy" or "difficult" according to each participant's unique performance pattern. As expected, the effect of the difficulty manipulation was preserved in these recoded data. An ANOVA of accuracy scores revealed a main effect of difficulty $F_{(1,17)}=9.36, p<0.05$. This effect was more pronounced in the perception condition, resulting in a task $\times$ difficulty interaction, $F_{(1,17)}=61.72, p<0.05$ (with no significant main effect of task). An examination of reaction times also revealed a main effect of difficulty, $F_{(1,17)}=15.16, p<0.05$, with no other effects present (Table 1). Critically, behavioral performance for the difficult condition of both tasks was matched in terms of accuracy and reaction times ( $t$ tests both $p>0.10$ ), allowing for direct comparison of BOLD activation without a difficulty confound. Additionally, accuracy for the luminance task did not differ from that of these two conditions.

\section{Imaging results}

First, we aimed to determine whether PRc was differentially involved in memory compared with our baseline task (i.e., luminance judgments). The contrast between all memory trials and all luminance trials revealed significant activation in the right PRc in the anterior collateral sulcus (peak $x=27, y=-4, z=-27 ; t=$ 4.01). Not surprisingly, this contrast also revealed activation in more posterior VVS regions, including the FFA (as defined with our functional localizer). Second, we determined whether PRc was also differentially involved in our perception task compared with baseline. The contrast between all perception trials and all luminance trials revealed significant activation in a right PRc region in the anterior collateral sulcus overlapping with that observed in the memory contrast (peak $x=33, y=-4, z=-26$; $t=4.20)$. Again, other more posterior VVS regions, including the FFA, were present in the pattern identified with this contrast. Supplemental Figure 2 (available at www.jneurosci.org as supplemental material) displays the overlap in activation for both contrasts for descriptive purposes. Notably, in itself the overlap in PRc does not address whether activation levels were of comparable magnitude or perhaps stronger for the memory task. Thus, we directly compared activation associated with accurate trials for the difficult memory and perception conditions, i.e., when performance was matched across tasks. This contrast revealed no significant activation in any aspect of PRc, even when the threshold was lowered to a level of $p=0.10$. Examination of the effects of the difficulty manipulation also showed no differential response in PRc.

In the next step of our analyses, we sought even more direct evidence for a comparable role of PRc in recognition memory and perceptual oddball decisions by focusing on accuracy effects. We asked whether activation in PRc is related to accuracy on both tasks. A conjunction analysis (Nichols et al., 2005) that combined an accuracy contrast for all memory trials with an accuracy contrast for all perception trials revealed a common activation in right PRc in the anterior collateral sulcus $(p<0.001 ; p<0.01$ for the individual contrasts; peak $x=18, y=5, z=-29$ ) (Fig. 2). Notably, the accuracy effect in PRc was not modulated by task difficulty in either task (i.e., interaction terms $>0.10$ ) (see Fig. 2 for response profile). Although not the specific focus of the current study, our conjunction contrast also revealed activity in a more lateral anterior temporal region in the left occipitotemporal sulcus (OTS; $p<0.001$, peak $x=-32, y=4, z=-37$ ). This finding is in line with other evidence pointing to an involvement of lateral inferior temporal lobe structures in making finegrained face discriminations (Kriegeskorte et al., 2007; Suzuki, 2009), and the notion that PRc does not perform its role in isolation.

To examine whether more posterior VVS regions in inferotemporal cortex showed more limited contributions to accuracy than PRc, we conducted a targeted ROI analysis of the functionally defined FFA. When investigating effects of task, accuracy and difficulty within a three-factorial $2 \times 2 \times 2$ ANOVA, we obtained a significant three-way interaction effect $\left[F_{(1,17)}=4.71, p<\right.$ $0.05]$. Subsequent pairwise comparisons revealed that this interaction reflected an accuracy effect that was limited to the easy perception condition $\left[t_{(17)}=3.35, p<0.05\right.$ Bonferroni corrected (Fig. 3)].

\section{Discussion}

In the present fMRI study, we compared the role of PRc in a forced-choice recognition memory task and in perceptual oddball judgments using highly similar, morphed face stimuli. Under conditions of matched levels of behavioral performance, we observed comparable levels of activation in right PRc for both tasks. Critically, right PRc activity also differentiated between accurate and inaccurate trials in both tasks. Together these results indicate 
that declarative memory demands are not a prerequisite for a performance-related engagement of PRc in object discriminations. Moreover, they show that the introduction of such declarative memory demands in an otherwise closely matched perceptual task does not lead to an increase in PRc involvement.

The observed accuracy effect in right PRc for the memory conditions converges with a large literature pointing to a critical role of PRc in recognition memory for objects and faces (Eichenbaum et al., 2007), including past neuroimaging findings showing accuracy effects in the context of yes-no recognition decisions (Gonsalves et al., 2005; Daselaar et al., 2006). Notably, in forced-choice recognition, unlike in yes-no recognition, previously studied items and novel foils are presented simultaneously. Thus, both familiarity of the target and novelty of the foils can guide successful discrimination. As the latter has typically led to higher levels of PRc activation in previous research, the pattern we observed could reflect a novelty signal for the foils that is more pronounced in the context of a correctly than incorrectly identified familiar target.

The accuracy effect for the perception task indicates that PRc contributes to perceptual discriminations of highly similar visual stimuli under conditions in which no reference to any prior encounter is required (Buckley and Gaffan, 2006; Murray et al., 2007). In fact, in the current study, no reference to any such prior encounter could be made; all stimuli in the perceptual task were novel and trial unique. Thus, neither familiarity nor novelty could be used as a source to distinguish between targets and foils. Accordingly, they cannot account for the observed differences in PRc activation for correct compared with incorrect trials.

Our perception task was modeled after the oddity task used by Buckley et al. (2001) to examine the effects of PRc lesions on visual discriminations in nonhuman primates under conditions of minimal declarative memory demands. These authors reported unimpaired performance in association with PRc lesions when the oddball was different in color, size, or simple shape. Yet, the lesions lead to impairments under conditions of high feature ambiguity, i.e., when faces and other complex visual stimuli that could not be distinguished based on simple perceptual features were presented. In related human patient research, large MTL lesions that include PRc have also been reported to produce impairments on oddball and other perceptual discrimination tasks with visual stimuli that share many shape features (Barense et al., 2005; Lee et al., 2005a,b; Barense et al., 2007). However, the findings are less intervals.
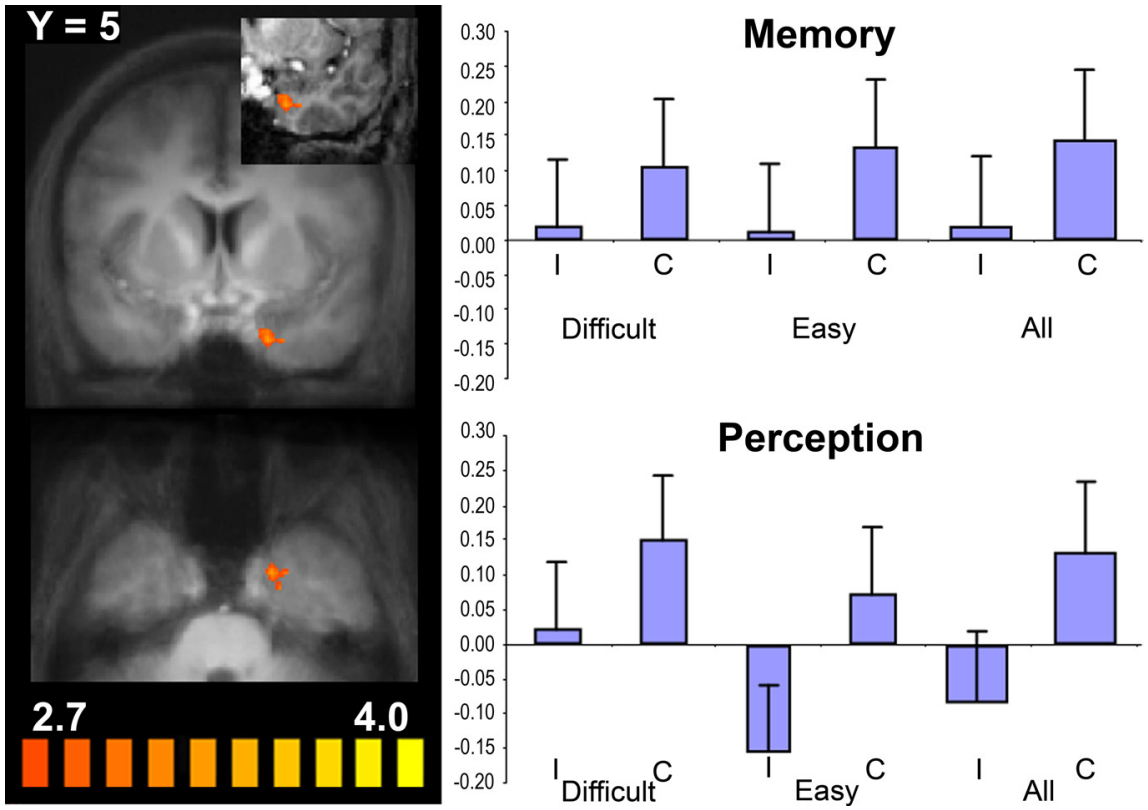

Figure 2. Coronal and transverse views of the right PRc region showing a differential response to accurate versus inaccurate trials on perception and memory tasks. Main images reflect MTL activation resulting from the conjunction analysis for accuracy effects in both tasks superimposed on the average structural MRI of all subjects within the range of $t$ values shown. Inset image reflects the same map superimposed on the structural MRI of a representative subject for purpose of visualization of the collateral sulcus. Beta weights for the cluster of voxels reaching our threshold for this contrast are presented for both tasks collapsed across difficulty levels and, for descriptive purposes, separately for each level. I, Incorrect; C, correct; error bars represent $95 \%$ confidence
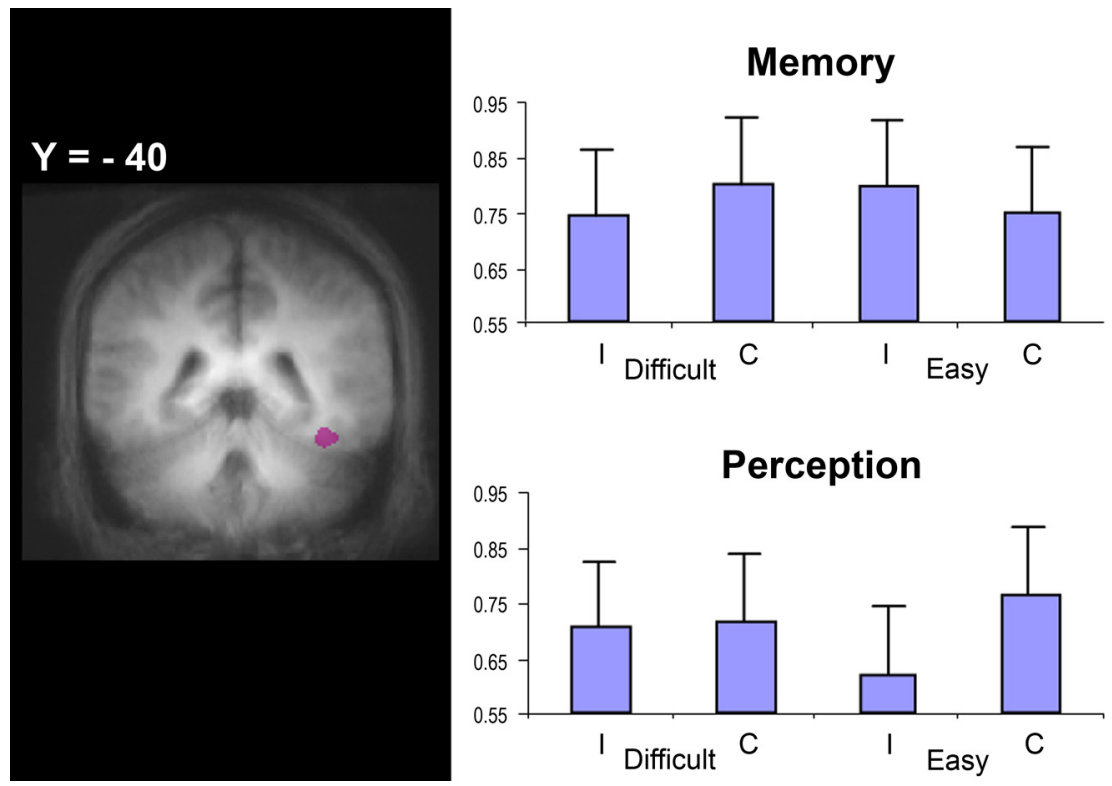

Figure 3. Coronal view of the right FFA region as defined by the independent functional localizer. Beta weights reflect responses in this region for the two difficulty conditions in each task. The accuracy effect is significant only for the easy perception condition. I, Incorrect; C, correct; error bars represent $95 \%$ confidence intervals.

consistent than those in the nonhuman primate literature, perhaps because of the variability in nature and extent of the lesions across studies (Levy et al., 2005; Shrager et al., 2006). Nevertheless, the findings reported by Lee et al. (2005a), who observed impairments in patients with large MTL lesions using morphed face stimuli very similar to those used here, are directly in line with our conclusions.

It has been proposed that PRc may reflect the highest level of 
object representation in the hierarchically organized VVS (Murray and Bussey, 1999; Bussey and Saksida, 2007). According to such a model, it is the nature of the representation required, rather than the presence or absence of declarative memory demands, that determines whether PRc is recruited in object discriminations. PRc may provide an object representation that is critical when individual shape features are insufficient for unique object identification. Although the present study was not designed to determine the exact nature of those object representations that require PRc contributions, comparison of activation in PRc and the more posterior FFA supports such a representational account. Unlike in PRc, accuracy effects observed in the FFA were specific to the easy perception condition, consistent with a more limited representational capability of more posterior regions in the VVS hierarchy (as theorized by the hierarchical representational account; also Kriegeskorte et al., 2007).

Curiously, accuracy effects in PRc were not modulated by difficulty of the perception task in the current study. These findings mirror a report of human patients with large MTL lesions who exhibited significant deficits in perceptual face discriminations independent of morph difficulty, despite observed difficulty effects for other classes of complex visual stimuli (Lee et al., 2005a). A large literature points to a critical role of holistic gestalt-like representations in face perception (Farah et al., 1998). Reliance on such a representation may be particularly critical when discriminations of morphed stimuli are required, as in the current study; morphing warps an image in nonlinear ways, making discriminations based on individual features unreliable (Cate and Köhler, 2006). Holistic representations are also thought to play a critical role in generalizing object or face identity across differences in viewpoint, a task demand critical in a number of past studies that have shown perceptual deficits in association with PRc lesions (Buckley and Gaffan, 2006). Although further research is necessary, such findings suggest that PRc may support gestalt-like visual representations. These representations would also be beneficial for recognition of prior occurrence, because they are relatively resistant to interference at the feature level from other encountered stimuli (Cowell et al., 2006).

Beyond representational demands, pre-experimental stimulus familiarity may also determine the extent of PRc contributions to perceptual discriminations. Functional neuroimaging research on working memory has shown that MTL regions in the vicinity of PRc are involved to a greater extent in the online maintenance of complex visual stimuli that are novel and lack pre-existing cortical representations than familiar ones (Stern et al., 2001). Accordingly, stimulus novelty may have also played a role in engaging PRc in perceptual discriminations in the current study. It has been suggested that rapid synaptic plasticity in PRc, critical for the formation of declarative memory representations, can also support the online maintenance of novel stimuli in working memory when posterior cortical regions are insufficient to perform this function (Ranganath et al., 2005; Hasselmo and Stern, 2006; Ranganath, 2006; Winters et al., 2008). The same mechanism may also support the online maintenance of novelface representations in difficult perceptual discriminations when there is no delay component and the stimuli are continuously visible. However, a follow-up experiment that we conducted to explore this idea at the behavioral level did not provide any support; it did not reveal any declarative-memory advantage for successfully identified oddball items on a subsequent recognition memory task (see supplemental material, available at www. jneurosci.org). Future research needs to address this issue more directly at the neural level to determine the extent to which PRc activity on perceptual oddball tasks may be related to rapid declarative-memory formation and/or working-memory maintenance.

In conclusion, our findings provide new evidence that the involvement of PRc in the accuracy of perceptual discriminations can match that in recognition decisions. Therefore, they show that the presence of declarative retrieval demands is neither a prerequisite nor a factor that necessarily boosts PRc engagement. As such our findings add to a growing literature indicating that declarative memory and perception are not as clearly separable at the level of MTL functioning as traditionally thought.

\section{References}

Barense MD, Bussey TJ, Lee AC, Rogers TT, Davies RR, Saksida LM, Murray EA, Graham KS (2005) Functional specialization in the human medial temporal lobe. J Neurosci 25:10239-10246.

Barense MD, Gaffan D, Graham KS (2007) The human medial temporal lobe processes online representations of complex objects. Neuropsychologia 45:2963-2974.

Baxter MG (2009) Involvement of medial temporal lobe structures in memory and perception. Neuron 61:667-677.

Buckley MJ, Gaffan D (2006) Perirhinal cortical contributions to object perception. Trends Cogn Sci 10:100-107.

Buckley MJ, Booth MC, Rolls ET, Gaffan D (2001) Selective perceptual impairments after perirhinal cortex ablation. J Neurosci 21:9824-9836.

Bussey TJ, Saksida LM (2007) Memory, perception, and the ventral visualperirhinal-hippocampal stream: thinking outside of the boxes. Hippocampus 17:898-908.

Cate AD, Köhler S (2006) The missing whole in perceptual models of perirhinal cortex. Trends Cogn Sci 10:396-397.

Cowell RA, Bussey TJ, Saksida LM (2006) Why does brain damage impair memory? A connectionist model of object recognition memory in perirhinal cortex. J Neurosci 26:12186-12197.

Daselaar SM, Fleck MS, Cabeza R (2006) Triple dissociation in the medial temporal lobes: recollection, familiarity, and novelty. J Neurophysiol 96:1902-1911.

Devlin JT, Price CJ (2007) Perirhinal contributions to human visual perception. Curr Biol 17:1484-1488.

Eichenbaum H, Yonelinas AP, Ranganath C (2007) The medial temporal lobe and recognition memory. Annu Rev Neurosci 30:123-152.

Farah MJ, Wilson KD, Drain M, Tanaka JN (1998) What is "special” about face perception? Psychol Rev 105:482-498.

Ganel T, Gonzalez CL, Valyear KF, Culham JC, Goodale MA, Köhler S (2006) The relationship between fMRI adaptation and repetition priming. Neuroimage 32:1432-1440.

Gonsalves BD, Kahn I, Curran T, Norman KA, Wagner AD (2005) Memory strength and repetition suppression: multimodal imaging of medial temporal cortical contributions to recognition. Neuron 47:751-761.

Hampton RR (2005) Monkey perirhinal cortex is critical for visual memory, but not for visual perception: reexamination of the behavioural evidence from monkeys. Q J Exp Psychol B 58:283-299.

Hasselmo ME, Stern CE (2006) Mechanisms underlying working memory for novel information. Trends Cogn Sci 10:487-493.

Insausti R, Juottonen $\mathrm{K}$, Soininen $\mathrm{H}$, Insausti AM, Partanen $\mathrm{K}$, Vainio P, Laakso MP, Pitkänen A (1998) MR volumetric analysis of the human entorhinal, perirhinal, and temporopolar cortices. Am J Neuroradiol 19:659-671.

Kanwisher N, McDermott J, Chun MM (1997) The fusiform face area: a module in human extrastriate cortex specialized for face perception. J Neurosci 17:4302-4311.

Kriegeskorte N, Formisano E, Sorger B, Goebel R (2007) Individual faces elicit distinct response patterns in human anterior temporal cortex. Proc Natl Acad Sci U S A 104:20600-20605.

Lee AC, Bussey TJ, Murray EA, Saksida LM, Epstein RA, Kapur N, Hodges JR, Graham KS (2005a) Perceptual deficits in amnesia: challenging the medial temporal lobe "mnemonic" view. Neuropsychologia 43:1-11.

Lee AC, Buckley MJ, Pegman SJ, Spiers H, Scahill VL, Gaffan D, Bussey TJ, Davies RR, Kapur N, Hodges JR, Graham KS (2005b) Specialization in the medial temporal lobe for processing of objects and scenes. Hippocampus 15:782-797. 
Lee AC, Scahill VL, Graham KS (2008) Activating the medial temporal lobe during oddity judgment for faces and scenes. Cereb Cortex 18:683-696.

Levy DA, Shrager Y, Squire LR (2005) Intact visual discrimination of complex and feature-ambiguous stimuli in the absence of perirhinal cortex. Learn Mem 12:61-66.

Milner B, Squire LR, Kandel ER (1998) Cognitive neuroscience and the study of memory. Neuron 20:445-468.

Murray EA, Bussey TJ (1999) Perceptual-mnemonic functions of the perirhinal cortex. Trends Cogn Sci 3:142-151.

Murray EA, Bussey TJ, Saksida LM (2007) Visual perception and memory: a new view of medial temporal lobe function in primates and rodents. Annu Rev Neurosci 30:99-122.

Nichols T, Brett M, Andersson J, Wager T, Poline JB (2005) Valid conjunction inference with the minimum statistic. Neuroimage 25:653-660.

Ranganath C (2006) Working memory for visual objects: complementary roles of inferior temporal, medial temporal, and prefrontal cortex. Neuroscience 139:277-289.

Ranganath C, Cohen MX, Brozinsky CJ (2005) Working memory mainte- nance contributes to long-term memory formation: neural and behavioral evidence. J Cogn Neurosci 17:994-1010.

Shrager Y, Gold JJ, Hopkins RO, Squire LR (2006) Intact visual perception in memory-impaired patients with medial temporal lobe lesions. J Neurosci 26:2235-2240.

Squire LR, Stark CE, Clark RE (2004) The medial temporal lobe. Annu Rev Neurosci 27:279-306.

Stern CE, Sherman SJ, Kirchhoff BA, Hasselmo ME (2001) Medial temporal and prefrontal contributions to working memory tasks with novel and familiar stimuli. Hippocampus 11:337-346.

Suzuki WA (2009) Perception and the medial temporal lobe: evaluating the current evidence. Neuron 61:657-666.

Suzuki WA, Amaral DG (1994) Perirhinal and parahippocampal cortices of the macaque monkey: cortical afferents. J Comp Neurol 350:497-533.

Winters BD, Saksida LM, Bussey TJ (2008) Object recognition memory: neurobiological mechanisms of encoding, consolidation and retrieval. Neurosci Biobehav Rev 32:1055-1070. 